\title{
ULTRACYTOCHEMISTRY OF STAMINAL HAIRS IN BULBINE SP.
}

\author{
Y. Naidoo, T. Xuma and G. Naidoo \\ School of Biological and Conservation Sciences, University of KwaZulu-Natal, \\ Private Bag X54001, Durban, 4000, South Africa
}

Staminal hairs occur in the flowers of several Angiosperm families. A broad survey of hairs on filaments of stamens has been recorded for the monocotyledons and the taxonomic value of these hairs, especially at the generic level is well established [1]. A wide range of different types of hairs are present in these species and there is much speculation on hair functions. However, very little information exists on the details of ultrastructure. The aim of this investigation is therefore to determine the ultrastructure of these hairs and also to suggest possible functions. For the present study the ultracytochemistry of the staminal hairs of Bulbine inflata oberm. and B. natalensis Bak., taken from flowers at different stages of development, was investigated. Each stamen is covered with hairs (Fig. 1), generally proximal to the anthers and on the outermost surface.

Flower buds from flowers of both species of Bulbine were removed from the inflorescence just before midday. The smallest buds were processed whole but larger buds were dissected and the stamens removed for processing. For SEM, stamens or whole buds were fixed for $2 \mathrm{~h}$ in $2.5 \%$ glutaraldehyde in $0.05 \mathrm{M} \mathrm{Na}$ cacodylate buffer, $\mathrm{pH} 7.0$ with a few drops of Triton X100 to facilitate wetting of the hairy stamens. Tissue was dehydrated in ethanol and critical-point dried from liquid $\mathrm{CO}_{2}$. Dried tissue was fixed to brass stubs with double-sided tape and coated with gold for $3 \mathrm{~min}$ at $20 \mathrm{kV}$. Samples were viewed in a Philips SEM 5000 at $12 \mathrm{kV}$. For TEM, stamens were fixed for $2 \mathrm{~h}$ in $2.5 \%$ glutaraldehyde, post fixed in $1 \%$ osmium tetroxide in $0.05 \mathrm{M} \mathrm{Na}$ cacodylate buffer for $2 \mathrm{~h}$, washed in water, stained en bloc in $2 \%$ aqueous uranyl acetate for $1 \mathrm{~h}$, dehydrated in ethanol, briefly immersed in propylene oxide and embedded in epoxy Spurr resin. Sections were stained in lead citrate for $2 \mathrm{~min}$ and viewed in a Philips TEM 301 at $80 \mathrm{kV}$. The cytochemistry of the staminal hairs was investigated using a variety of staining techniques. These included osmium ferricyanide staining, zinc iodide osmium impregnation, silver hydroxylamine and aldehyde localization.

The hairs arise as single cell outgrowths from the epidermis of the filament and appear to be fully developed in all but the very young green buds. The surface of each hair is raised in anticlockwise helical wall thickenings (Fig. 2). The walls consist of several clearly distinguishable layers and the cytoplasm is well-developed with a large central vacuole forming as the flowers begin to open. The cytoplasm contains mitochondria and ribosomes but few dictyosomes. The cytoplasm of both the hair and the epidermis of the filament contain plastids with large starch grains and peripheral droplets, possibly of a lipid nature (Fig. 3). These droplets react with ammoniacal silver hydroxylamine indicating the presence of ester linkages. Postfixation with osmium ferricyanide revealed strands of tubular smooth endoplasmic reticulum (SER) running along the length of the filament (Fig. 4). The SER appear to surround/anastomize each plastid in the hair.

The distinct presence of endoplasmic reticulum suggests that the hairs may have a secretory function but the nature of the secretion has not been elucidated. 
References

[1] R. M. T. Dahlgren and H. T. Clifford, Monocotyledons: A comparative study. Academic Press, London, 1982.
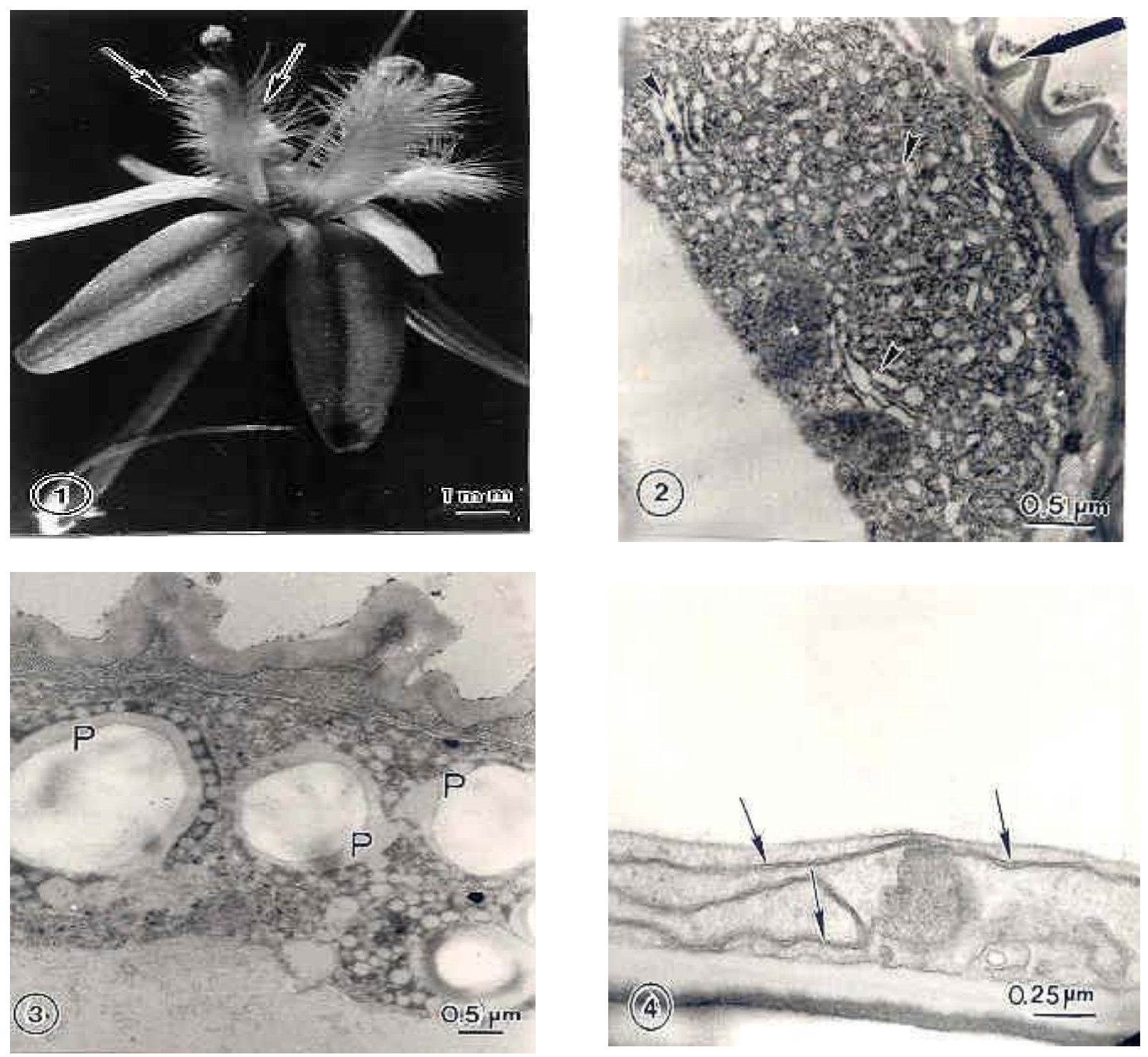

Fig. 1. Open flower of Bulbine inflata showing long hairs \{arrows\} on each filament.

Fig. 2. Hair cell showing helical wall thickenings arrow $\}$ and bundles of endoplasmic reticulum in cytoplasm \{arrowheads\}.

Fig. 3. Hair cell from $B$. inflata showing plastids (P) and general ultrastructure.

Fig. 4. Osmium ferricyanide staining reveals smooth endoplasmic reticulum \{arrows running along length of filament. 